\title{
Stochastic Consensus of Single-Integrator Multiagent Systems with Inherent Nonlinear Dynamics and Measurement Noises in Directed Fixed Topologies
}

\author{
Sabir Djaidja and Qinghe Wu \\ School of Automation, Beijing Institute of Technology, Hai Dian District, Beijing 100081, China \\ Correspondence should be addressed to Sabir Djaidja; djaidjasaber@yahoo.fr
}

Received 27 July 2014; Accepted 18 November 2014; Published 7 December 2014

Academic Editor: Ramachandran Raja

Copyright (c) 2014 S. Djaidja and Q. Wu. This is an open access article distributed under the Creative Commons Attribution License, which permits unrestricted use, distribution, and reproduction in any medium, provided the original work is properly cited.

Consensus of continuous-time single-integrator multiagent systems with inherent nonlinear dynamics and measurement noises is addressed in this paper. The consensus controller is developed for directed interaction topologies. Each agent's control input is constructed based on its own state and its neighbors' states corrupted by noises. The control input contains a time-varying consensus gain in order to attenuate the noises. Conditions for ensuring mean square convergence under noisy measurement and asymptotic convergence in the noise-free case are derived. Finally, some simulations were carried out to show the effectiveness of our control law and how it can solve the consensus problem.

\section{Introduction}

Over the last few years, there has been a considerable interest in the study of distributed cooperative control in multiagent systems. On one hand, this is mainly due to the advantages provided by cooperative control such as higher robustness, efficiency, accuracy, and speed in accomplishing tasks in comparison to single agent. On other hand, it is because of its widespread applications in real world such as exploration [1], mapping of unknown or partially known environment [2], distributed sensor fusion and localization [3], transportation [4], and hazardous cleaning labors [5].

As a fundamental research field in distributed cooperative control, consensus becomes an attractive one. Consensus simply means a group of agents exchanging information between them to reach some common states; under this definition, consensus can be classified into two main categories: leaderless consensus in the case when there is no group reference trajectory known by the team's members $[6,7]$ and leader-following consensus when a virtual or physical leader exists in the team [8-10].

An important point can be remarked on the distributed consensus controllers mentioned in [6-10] and most of references therein, that the inherent nonlinearity is not considered in the agent's model. In fact, inherent nonlinear dynamics exists in wide class of practical systems, such as friction characterizing the dynamics in the absence of any control input. Inherent nonlinear dynamics was well studied from the aspect of synchronization in complex networks [11] and references therein. Recently, inherent nonlinear dynamics received an intensive attention in the studies of consensus problem. References [12, 13] studied the consensus in directed networks for single-integrators agents with inherent dynamics. Reference [14] studied a finite time consensus for single-integrator under undirected topologies. Reference [15] proposed a finite time consensus control for single-integrator kinematics with unknown inherent nonlinear dynamics under directed interaction topologies. Second-order consensus with inherent nonlinear dynamics under undirected topology was handled in [16], and second-integrators dynamics with inherent nonlinear dynamics under directed topologies was also investigated in $[13,17]$. In [18], the higher-order dynamics with inherent nonlinear dynamics was addressed.

The above-mentioned references which deal with inherent nonlinear dynamics showed the necessary and sufficient conditions under which the consensus is reached, and 
the final consensus value depends on initial values or satisfies certain dynamics. To regulate the final states of agents on a desired one, some consensus tracking controllers were developed to make a group of agents converge to a common destination or follow a predefined reference trajectory. Reference [19] investigated the consensus tracking for singleintegrator dynamics with inherent nonlinear dynamics under fixed communication topology, and tracking consensus was achieved only with the position's measurement of the neighbors and the virtual leader. In [20] and based on sliding mode theory, a finite time consensus tracking for single-integrator dynamics with inherent nonlinear under fixed topologies was addressed, with the constraint that all the followers should have access to the leader. Reference [21] proposed a secondorder leader-following consensus of nonlinear multiagent systems via pinning control in which some conditions were established to define which kind of agent and how many agents should be pinned to guarantee that the whole group will track the leader. Reference [22] proposed a connectivitypreserving second-order consensus algorithm with virtual leader. Besides consensus tracking for single and double integrator, cooperative tracking for higher-order nonlinear systems was also studied in the literature. Reference [23] proposed an adaptive cooperative tracking for higher-order nonlinear systems with unknown dynamics and external disturbance.

In addition to the existence of inherent nonlinearities, another major problem can be met in multiagent systems that the communication between agents is often performed in an uncertain environment, which means that the communication suffers always from the noises. Over the last few years, some references addressed the problem of consensus under additive measurement noises for both leaderless and leaderfollowing cases [24-33].

For the leaderless case, [24] introduced a decreasing consensus gain to attenuate the measurement noises for firstorder discrete-time in strongly connected circulant graph. References $[25,26]$ extended the work done in [24] to the strongly connected digraphs and digraphs having a spanning tree, respectively. Switching topology for first-order discretetime was addressed in [27], where sufficient conditions for mean square average consensus and almost sure consensus are given. Reference [28] derived the necessary and sufficient conditions on the consensus gain under which first-order continuous-time achieves asymptotic unbiased mean square average consensus under fixed and directed topologies. In [29], first-order continuous-time under measurement noises and time-varying communication delay has been addressed. Necessary and sufficient conditions for consensus of doubleintegrator multiagent systems with measurement noises under fixed topologies have been derived in [30].

For the leader-following case, [31, 32] used a velocity decomposition technique to design a neighbor-based tracking protocol and a distributed estimator to achieve mean square convergence for leader-following multiagent system under measurement noises in both directed fixed and timevarying topologies. Reference [33] proposed a consensus control for leader-following single-integrator multiagent systems and conditions to ensure the strong mean square convergence in directed and switching topologies were obtained. Reference [34] derived conditions under which the leaderfollowing consensus of double-integrator consensus with constant velocity reference state is achieved in mean square for both directed fixed and switching topologies.

Recently, some researchers have addressed the consensus problem of multiagent systems under multiplicative measurement noises [35-37], where the noises' intensities are considered proportional to the relative states.

Based on the above background, one can see that the problems of consensus with inherent nonlinear dynamics and consensus with measurement noises have been studied separately. In fact, the two constraints can exist together in one multiagent systems, when the dynamic model of the agent contains an inherent nonlinear part and at the same time the measured neighbors' states by this agent present an additive noise. Our main contributions in this paper are as follows. First, we propose a unified cooperative consensus controller for single-integrator multiagent systems with inherent nonlinear dynamics and measurement noises under directed fixed topologies. For our knowledge, there are no references, which study the consensus under these constraints in a unified cooperative controller. Second, by the end of this paper, we will deduce that our proposed control law in the absence of the inherent nonlinear dynamics can ensure mean square convergence in digraphs having spanning tree; this result is more general compared to that achieved in [28] for balanced digraphs.

The remainder of this paper is organized as follows. Some preliminaries and definitions are given in Section 2. Then, the problem is formulated in Section 3. Section 4 states the main results. Section 5 is devoted to confirm the effectiveness of the control proposed through some simulations. Finally, Section 6 concludes this paper.

\section{Background}

Information exchange between vehicles can be modeled by directed or undirected graphs. A digraph (directed graph) consists of a pair $(\mathcal{N}, \mathscr{E})$, where $\mathcal{N}$ is a finite nonempty set of nodes and $\mathscr{E} \in \mathscr{N} \times \mathscr{N}$ is a set of ordered pairs of nodes, called edges. An edge $(i, j)$ in a digraph denotes that vehicle $j$ can obtain information from vehicle $i$, but not necessarily vice versa.

The adjacency matrix $\mathscr{A}=\left[a_{i j}\right] \in \mathfrak{R}^{n \times n}$ of a weighted digraph is defined as $a_{i i}=0$ and $a_{i j}>0$ if $(j, i) \in \mathscr{E}$, where $i \neq j$. The adjacency matrix of a weighted undirected graph is defined accordingly except that $a_{i j}=a_{j i}, \forall(i \neq j)$, since $(j, i) \in \mathscr{E}$ implies $(i, j) \in \mathscr{E}$. Let matrix $L=\left[l_{i j}\right] \in \mathfrak{R}^{n \times n}$ be defined as $l_{i i}=\sum_{j \neq i} a_{i j}$ and $l_{i j}=-a_{i j}$, where $i \neq j$.

Matrix $L$ satisfies the following conditions:

(i) $l_{i j} \leq 0, i \neq j$;

(ii) $\sum_{j=1}^{n} l_{i j}=0, i=1, \ldots, n$.

For an undirected graph, $L$ is called the Laplacian matrix, which has the property that it is symmetric positive semidefinite. However, $L$ for a digraph does not have this property. 
A directed path is a sequence of ordered edges of the form $\left(\mathscr{V}_{i 1}, \mathscr{V}_{i 2}\right),\left(\mathscr{V}_{i 2}, \mathscr{V}_{i 3}\right), \ldots$, where $\mathscr{V}_{i j} \in \mathcal{N}$, in a digraph.

A digraph is called strongly connected, if there is a directed path from every node to every other node. An undirected graph is called connected, if there is a path between any distinct pair of nodes.

A directed spanning tree of a digraph is a directed tree formed by graph edges that connect all of the nodes of the graph. A graph has (or contains) a directed spanning tree, if there exists a directed spanning tree, being a subset of the graph. Note that the condition that a digraph has a directed spanning tree is equivalent to the case that there exists at least one node having a directed path to all of the other nodes. In the case of undirected graphs, having an undirected spanning tree is equivalent to being connected. However, in the case of directed graphs, having a directed spanning tree is a weaker condition than being strongly connected [38].

Definition 1 (see [39] ( $P$ uniformly decreasing)). Given a positive symmetric matrix $P$, there exists a matrix $Q$ such that the vector field $f(t, x)-Q x$ is $P$ uniformly decreasing; that is,

$$
\begin{aligned}
& \left(x_{1}-x_{2}\right)^{T} P\left[f\left(t, x_{1}\right)-Q x_{1}-f\left(t, x_{2}\right)+Q x_{2}\right] \\
& \quad \leq-l\left|x_{1}-x_{2}\right|^{2},
\end{aligned}
$$

for some $l>0$ and all $x_{1}, x_{2} \in \mathfrak{R}^{n}$.

Definition 2. A matrix $A(t): t \rightarrow R^{n \times n}$ is said to be uniformly negative definite, and one writes $(A(t) \ll 0)$, if $\exists \varepsilon>$ $0:\langle A(t) x, x\rangle \leq-\varepsilon x^{2}<0, \forall x \in R^{n}, t \geq 0$, and it can also be written in a simplified form: $A(t) \leq-\varepsilon I<0$.

\section{Problem Formulation}

Consider a multiagent system with inherent nonlinear dynamics composed of $n$ autonomous agents described by single-integrator dynamics

$$
\dot{x}_{i}=u_{i}+f\left(t, x_{i}\right), \quad i=1, \ldots, n,
$$

where $x_{i} \in \mathfrak{R}^{m}, u_{i} \in \mathfrak{R}^{m}$ are, respectively, the state and the control input of the ith agent. $f\left(t, x_{i}\right) \in \mathfrak{R}^{m}$ is a nonlinear vector-valued continuous function that represents the unknown inherent nonlinear dynamics. For simplicity, in the remainder of this paper, we let $m=1$.

The $i$ th agent receives information from its neighbors with measurement noises:

$$
x_{j i}^{*}=x_{j}(t)+\sigma_{j i} \eta_{j i}(t),
$$

where $x_{j i}^{*}(t)$ is the measurement of the $j$ th agent's state by the $i$ th agent, $n_{j i}(t)$ is a standard white noise, and $\sigma_{j i} \geq 0$ is the noise intensity.

Here, we note that in the real physical world, an agent can get its neighbors' states information in two ways. The first one is that the agent measures directly its neighbors' states by using some sensors. However, this technique is restrictive and hard. In this case, the noises corrupting the information are due to the sensors themselves, and these noises are known by measurement noises. The second way is that each agent senses its own states and transmits it to agents that have access to him. This second technique is the most practical one. The noises involved here are due to the transmission process and they are known by communication noises. In many literatures, the two notions measurement and communication are interchangeable.

In this paper, we aim to design a consensus protocol for system (2) under directed fixed topologies to make the agents' states converge in weak mean square; that is, $\lim _{t \rightarrow \infty} E\left[x_{i}(t)-\right.$ $\left.x_{j}(t)\right]^{2}=0$. And if the inherent dynamics is linear in $x$, then this consensus protocol ensures that each agent converges in mean square to a common random value $y^{*}$; that is, $\lim _{t \rightarrow \infty} E\left[x_{i}(t)-y^{*}\right]^{2}=0$.

The following notations will be used throughout this paper. $I_{n}$ denotes the $n \times n$ dimensional identity matrix. $1_{n}$ denotes the $n$-dimensional one vector. For a vector or matrix $A, A^{T}$ denotes its transpose; if $A$ is a matrix, then $\operatorname{tr}(A)$ denotes its trace and $\lambda_{\max }(A)$ and $\lambda_{\min }(A)$, its maximum and minimum eigenvalues, respectively. For a given random variable $X, E[X]$ denotes its mathematical expectation.

For the construction of our consensus protocol, we need the following two assumptions.

Assumption 3. The topology among the $n$ agents $\mathscr{G}(\mathscr{V}, \mathscr{E}, \mathscr{A})$ has a spanning tree.

Assumption 4. $f(t, x)-Q x$ is $P$ uniformly decreasing.

Remark 5. The $P$ uniformly decreasing functions are widely used in the literature in the studying of synchronization of coupled network systems [40-42]. The condition of $P$ uniformly decreasing for a real matrix $P>0$ implies that $\dot{x}=f(t, x)$ is globally asymptotically stable. In particular, if $f(t, x)-Q x$ is $P$ uniformly decreasing for some real matrix $P>0$, the matrix $Q$ has a quite similar role to that of the Lipschitz constant in Lipschitz continuous functions; it bounds the inherent nonlinear dynamics and plays an important role in the stability of the system, and the term $-Q x$ can be seen as a linear feedback stabilizing the inherent nonlinear dynamics $f(t, x)$. If the inherent nonlinear dynamics $f(t, x)$ is asymptotically stable, then matrix $Q$ could be negative definite matrix. Otherwise, $Q$ could be positive definite.

\section{Consensus Protocol}

For the $i$ th agent, we propose the following consensus controller:

$$
u_{i}(t)=\alpha(t)\left[\sum_{j \in \mathcal{N}_{i}} a_{i j}\left(x_{j i}^{*}(t)-x_{i}(t)\right)\right], \quad i=1,2, \ldots, n,
$$

where $\alpha(t):[0, \infty) \rightarrow(0, \infty)$, called time-varying consensus gain, is a piecewise continuous differentiable function satisfying $\int_{0}^{\infty} \alpha(t) d t=\infty, \int_{0}^{\infty} \alpha^{2}(t) d t<\infty$, and $\lim _{t \rightarrow \infty} \alpha(t)=$ 0 and $\mathcal{N}_{i}=\mathcal{N}_{i}(\mathscr{G})$ denotes the neighbor set of the agent $i$. 
For the time-varying consensus gain, one can choose it as a function from the class $\alpha(t)=1 /(q+t)^{\delta}$, where $q>0$ and $0.5<\delta \leq 1$. This class of functions satisfies all the above conditions.

Substituting (4) into (2) and replacing $x_{j i}^{*}(t)$ by its expression from (3) give the following:

$$
\begin{array}{r}
\dot{x}_{i}(t)=f\left(t, x_{i}\right)+\alpha(t)\left[\sum_{j \in \mathcal{N}_{i}} a_{i j}\left(x_{j}(t)+\sigma_{j i} \eta_{j i}(t)-x_{i}(t)\right)\right], \\
i=1,2, \ldots, n .
\end{array}
$$

We define the matrix $M=\operatorname{diag}\left[\beta_{1} \Sigma_{1}, \beta_{2} \Sigma_{2}, \ldots, \beta_{n} \Sigma_{n}\right]$, where $\beta_{i}=\left[a_{i 1}, a_{i 2}, \ldots, a_{i n}\right]$ is the $i$ th row of the adjacency matrix $\mathscr{A}$ and $\Sigma_{i}=\operatorname{diag}\left[\sigma_{1 i}, \sigma_{2 i}, \ldots, \sigma_{n i}\right]$, and we define the vector $\eta(t)=$ $\left[\eta_{1}^{T}(t), \eta_{2}^{T}(t), \ldots, \eta_{n}^{T}(t)\right]^{T}$, where $\eta_{i}(t)=\left[\eta_{1 i}(t), \eta_{2 i}(t), \ldots\right.$, $\left.\eta_{n i}(t)\right]^{T}$.

System (5) can be rewritten in matrix form as follows:

$$
\dot{X}(t)=F(t, X)+\alpha(t)(-L) X(t)+\alpha(t) M \eta(t),
$$

where $X(t)=\left[x_{1}^{T}(t), x_{2}^{T}(t), \ldots, x_{n}^{T}(t)\right]^{T}$ and $F(t, X)=$ $\left[f\left(t, x_{1}\right)^{T}, f\left(t, x_{2}\right)^{T}, \ldots, f\left(t, x_{n}\right)^{T}\right]^{T}$.

We will introduce a variable transformation $S$ defined as

$$
Y(t)=S X(t)
$$

where $S$ is the matrix transformation given by

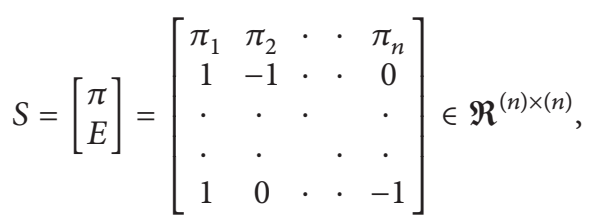

with $\pi=\left[\pi_{1}, \pi_{2}, \ldots, \pi_{n}\right]$ being the normalized first left eigenvector of the Laplacian matrix $L$ associated with the zero eigenvalue. Thus, $\pi L=0$ and $\sum_{1}^{n} \pi_{i}=1$.

We define also the vector $Y(t)$ as $Y(t)=\left[\pi X(t), Y_{e}^{T}(t)\right]^{T}$, where

$$
\begin{aligned}
Y_{e}(t) & =\left[y_{2}(t), y_{3}(t), \ldots, y_{n}(t)\right]^{T}=E X(t) \\
& =\left[x_{1}(t)-x_{2}(t), x_{1}(t)-x_{3}(t), \ldots, x_{1}(t)-x_{n}(t)\right]^{T} .
\end{aligned}
$$

It is clear that $S$ is nonsingular and its inverse has the form $S^{-1}=\left[\begin{array}{ll}1_{n} & R\end{array}\right]$.

By applying this transformation, (6) can be written with respect to $Y(t)$ as follows:

$$
\dot{Y}(t)=S F(t, X)+\alpha(t) S(-L) S^{-1} Y(t)+\alpha(t) S M \eta(t) .
$$

In first time, let us denote by $y_{1}(t)=x_{1}(t)$, and then

$$
\begin{gathered}
f\left(t, x_{1}\right)-f\left(t, x_{i}\right)=f\left(t, y_{1}\right)-f\left(t, y_{1}-y_{i}\right), \quad i=2, \ldots, n, \\
S F(t, X)=\left[\pi F(t, X), f\left(t, y_{1}\right)-f\left(t, y_{1}-y_{2}\right), \ldots,\right. \\
\left.f\left(t, y_{1}\right)-f\left(t, y_{1}-y_{n}\right)\right]^{T} \\
=\left[\pi F(t, X), F_{e}\left(t, Y_{e}\right)^{T}\right]^{T} .
\end{gathered}
$$

Equation (10) can be divided into two subsystems as follows:

$$
\begin{gathered}
\pi \dot{X}(t)=\pi F(t, X)+\alpha(t) \pi M \eta(t) \\
\dot{Y}_{e}(t)=F_{e}\left(t, Y_{e}\right)-\alpha(t) E L R Y_{e}(t)+\alpha(t) E M \eta(t) .
\end{gathered}
$$

In the Itô stochastic differential form, this can be written as

$$
\begin{gathered}
\pi d X(t)=\pi F(t, X) d t+\alpha(t) \pi M d W(t), \\
d Y_{e}(t)=F_{e}\left(t, Y_{e}\right) d t-\alpha(t) E L R Y_{e}(t) d t+\alpha(t) \operatorname{EMdW}(t),
\end{gathered}
$$

where $W=\left[W_{11}, \ldots, W_{n 1}, W_{12}, \ldots, W_{n 2}, \ldots, W_{1 n}, \ldots, W_{n n}\right]^{T}$ are independent Brownian motions.

Theorem 6. If Assumptions 3 and 4 hold, for the singleintegrator multiagent systems (2), the consensus protocol (4) asymptotically achieves weak mean square convergence under directed fixed topologies for any initial condition, if the following condition is satisfied:

$$
-2 l I_{n-1}+2 P Q-\alpha(t) I_{n-1} \ll 0 .
$$

Moreover, if $f(t, x)$ is linear in $x$, the consensus protocol (4) asymptotically achieves mean square convergence.

Proof. This proof will pass by two steps. First, we will prove that all the agents' states in the team converge in weak mean square. Second, we will show that if the inherent nonlinear dynamics $f(t, x)$ is linear in $x$, the agents' states converge in mean square to a random variable $y^{*}$.

Choose the Lyapunov function candidate as

$$
V(t)=Y_{e}^{T}(t) P Y_{e}(t)
$$

where $P \in \mathfrak{R}^{(n-1) \times(n-1)}$ is positive definite matrix that satisfies

$$
(-E L R) P+P(-E L R)^{T}=-I_{n-1} \text {. }
$$

We have $S F(t, X)=\left[\pi F(t, X), f\left(t, x_{1}\right)-f\left(t, x_{2}\right), \ldots\right.$, $\left.f\left(t, x_{1}\right)-f\left(t, x_{n}\right)\right]^{T}$. 
By applying the Itô formula [43], we get

$$
\begin{aligned}
d V(t)= & 2 Y_{e}^{T}(t) P\left[F_{e}\left(t, Y_{e}\right)+Q Y_{e}(t)-Q Y_{e}(t)\right] d t \\
& +\alpha(t) Y_{e}^{T}(t)\left[(-E L R) P+P(-E L R)^{T}\right] Y_{e}(t) d t \\
& +\alpha^{2}(t) \operatorname{tr}\left(P(E M)(E M)^{T}\right) d t \\
& +2 \alpha(t) Y_{e}^{T}(t) P E M d W(t), \\
d V(t)= & 2 Y_{e}^{T}(t) P\left[F_{e}\left(t, Y_{e}(t)\right)-Q Y_{e}(t)\right] d t \\
& +2 Y_{e}^{T} P Q Y_{e}(t) d t+\alpha(t) Y_{e}^{T}(t) \\
& \times\left[(-E L R) P+P(-E L R)^{T}\right] Y_{e}(t) d t \\
& +\alpha^{2}(t) \operatorname{tr}\left(P(E M)(E M)^{T}\right) d t \\
& +2 \alpha(t) Y_{e}^{T}(t) P E M d W(t) .
\end{aligned}
$$

Using Assumption 4, $2 Y_{e}^{T}(t) P\left[F_{e}\left(t, Y_{e}\right)-Q Y_{e}(t)\right] \leq$ $-2 l Y_{e}^{T}(t) Y_{e}(t)$, and, by Assumption 3, our digraph has a spanning tree; thus, $L$ has one zero eigenvalue and all the other eigenvalues are in the right half plane and because $S(L) S^{-1}=\left[\begin{array}{cc}0 & 0_{1 \times(n-1)} \\ 0_{(n-1) \times 1} & E L R\end{array}\right]$ is a congruence transformation preserving the number and the sign of eigenvalues. Hence, all the eigenvalues of (ELR) are in the right half plane and the matrix $(-E L R)$ is a Hurwitz stable matrix, and we can choose

$$
(-E L R) P+P(-E L R)^{T}=-I_{n-1} \text {. }
$$

Then,

$$
\begin{aligned}
d V(t) \leq & Y_{e}^{T}(t)\left[-2 l I_{n-1}+2 P Q-\alpha(t) I_{n-1}\right] Y_{e}(t) d t \\
& +\alpha^{2}(t) \operatorname{tr}\left(P(E M)(E M)^{T}\right) d t \\
& +2 \alpha(t) Y_{e}^{T}(t) P E M d W(t)
\end{aligned}
$$

Furthermore, we have $Y_{e}^{T}(t) P Y_{e}(t) \leq \lambda_{\max }(P) Y_{e}^{T}(t) Y_{e}(t)$. Then,

$$
\begin{aligned}
d V(t) \leq & {\left[-2 l I_{n-1}+2 P Q-\alpha(t) I_{n-1}\right] \frac{V(t)}{\lambda_{\max }(P)} d t } \\
& +\alpha^{2}(t) \operatorname{tr}\left(P(E M)(E M)^{T}\right) d t \\
& +2 \alpha(t) Y_{e}^{T}(t) P E M d W(t) .
\end{aligned}
$$

Denote by $D^{+} V(t)=(E[V(t+h)]-E[V(t)]) / h$, and note that, by choosing a proper stopping time, we can prove that the mathematical expectation $E \int_{t_{0}}^{t} \alpha(s) Y_{e}^{T}(t) P E M d W(s)=0$ [28, Lemma 3.1]. This implies

$D^{+} V(t)$

$$
\begin{aligned}
\leq & \frac{\left(1 / \lambda_{\max }(P)\right) \int_{t}^{t+h}\left(-2 l I_{n-1}+2 P Q-\alpha(s) I_{n-1}\right) E[V(s)] d s}{h} \\
& +\frac{\operatorname{tr}\left(P(E M)(E M)^{T}\right) \int_{t}^{t+h} \alpha^{2}(s) d s}{h}
\end{aligned}
$$

$\lim _{h \rightarrow 0^{+}} \sup D^{+} V(t)$

$$
\begin{aligned}
& \leq \lim _{h \rightarrow 0^{+}} \sup \left(\left(\frac{1}{\lambda_{\max }(P)} \int_{t}^{t+h}\left(-2 l I_{n-1}+2 P Q-\alpha(s) I_{n-1}\right)\right.\right. \\
& \left.\quad \times E[V(s)] d s) \times h^{-1}\right) \\
& +\lim _{h \rightarrow 0^{+}} \sup \frac{\operatorname{tr}\left(P(E M)(E M)^{T}\right) \int_{t}^{t+h} \alpha^{2}(s) d s}{h} \\
& =\left(-2 l I_{n-1}+2 P Q-\alpha(t) I_{n-1}\right) E[V(t)] \\
& \quad+\operatorname{tr}\left(P(E M)(E M)^{T}\right) \alpha^{2}(t) .
\end{aligned}
$$

If $-2 l I_{n-1}+2 P Q-\alpha(t) I_{n-1} \ll 0$, it implies that there exists an $\varepsilon>0$, such that $-2 l I_{n-1}+2 P Q-\alpha(t) I_{n-1}<-\varepsilon I_{n-1}$; hence, (22) becomes

$$
\begin{aligned}
\lim _{h \rightarrow 0^{+}} \sup D^{+} V(t) \leq & -\varepsilon E[V(t)] \\
& +\operatorname{tr}\left(P(E M)(E M)^{T}\right) \alpha^{2}(t) .
\end{aligned}
$$

By the comparison principle [44, Theorem 2.6.4],

$$
\begin{aligned}
E[ & V(t)] \\
\leq & E[V(0)] \exp \left\{\frac{1}{\lambda_{\max }(P)} \int_{0}^{t}(-\varepsilon) d s\right\} \\
& +\operatorname{tr}\left(P(E M)(E M)^{T}\right) \exp \left\{\frac{1}{\lambda_{\max }(P)} \int_{0}^{t}(-\varepsilon) d \xi\right\} \\
& \times \int_{0}^{t} \alpha^{2}(s) \exp \left\{\frac{1}{\lambda_{\max }(P)} \int_{0}^{t}(\varepsilon) d \xi\right\} d s \\
= & E[V(0)] \exp \left\{\frac{1}{\lambda_{\max }(P)} \int_{0}^{t}(-\varepsilon) d s\right\} \\
& +\operatorname{tr}\left(P(E M)(E M)^{T}\right) \\
& \times \int_{0}^{t} \alpha^{2}(s) \exp \left\{\frac{1}{\lambda_{\max }(P)} \int_{s}^{t}(-\varepsilon) d \xi\right\} d s .
\end{aligned}
$$

Here, following a similar proof in [33], we can prove that the second term in (24) equals zero when $t \rightarrow \infty$. Denote

$$
\begin{aligned}
I= & \operatorname{tr}\left(P(E M)(E M)^{T}\right) \\
& \times \int_{0}^{t} \alpha^{2}(s) \exp \left\{\frac{1}{\lambda_{\max }(P)} \int_{s}^{t}(-\varepsilon) d \xi\right\} d s .
\end{aligned}
$$


By the property that $\int_{0}^{\infty} \alpha^{2}(t) d t<\infty$, that implies for any given $c>0$ there exists $s_{0}>0$ such that $\int_{s_{0}}^{\infty} \alpha^{2}(s) d s<c$ and $I$ can be rewritten as

$$
\begin{aligned}
I= & \operatorname{tr}\left(P(E M)(E M)^{T}\right) \\
& \times \int_{0}^{s_{0}} \alpha^{2}(s) \exp \left\{\frac{1}{\lambda_{\max }(P)} \int_{s}^{t}(-\varepsilon) d \xi\right\} d s \\
& +\operatorname{tr}\left(P(E M)(E M)^{T}\right) \\
& \times \int_{s_{0}}^{t} \alpha^{2}(s) \exp \left\{\frac{1}{\lambda_{\max }(P)} \int_{s}^{t}(-\varepsilon) d \xi\right\} d s .
\end{aligned}
$$

Notice that when $0 \leq s \leq s_{0}$

$$
\exp \left\{\frac{1}{\lambda_{\max }(P)} \int_{s}^{t}(-\varepsilon) d \xi\right\} \leq \exp \left\{\frac{1}{\lambda_{\max }(P)} \int_{s_{0}}^{t}(-\varepsilon) d \xi\right\} .
$$

Notice also that

$$
\exp \left\{\frac{1}{\lambda_{\max }(P)} \int_{s}^{t}(-\varepsilon) d \xi\right\}<1 .
$$

Using (27) and (28) in the two terms of $I$, respectively, yields

$$
\begin{aligned}
I \leq & \operatorname{tr}\left(P(E M)(E M)^{T}\right) \exp \left\{\frac{1}{\lambda_{\max }(P)} \int_{s_{0}}^{t}(-\varepsilon) d \xi\right\} \\
& \times \int_{0}^{s_{0}} \alpha^{2}(s) d s+\operatorname{tr}\left(P(E M)(E M)^{T}\right) \int_{s_{0}}^{t} \alpha^{2}(s) d s \\
\leq & \operatorname{tr}\left(P(E M)(E M)^{T}\right) \exp \left\{\frac{1}{\lambda_{\max }(P)} \int_{s_{0}}^{t}(-\varepsilon) d \xi\right\} \\
& \times \int_{0}^{\infty} \alpha^{2}(s) d s+\operatorname{tr}\left(P(E M)(E M)^{T}\right) \int_{s_{0}}^{\infty} \alpha^{2}(s) d s .
\end{aligned}
$$

By choosing a small enough $c$ and because $\lim _{t \rightarrow \infty} \exp \{(1 /$ $\left.\left.\lambda_{\text {max }}(P)\right) \int_{s_{0}}^{t}(-\varepsilon) d \xi\right\}=0, \lim _{t \rightarrow \infty} I=0$.

In addition, it is straightforward to show that

$$
\lim _{t \rightarrow \infty} E[V(0)] \exp \left\{\frac{1}{\lambda_{\max }(P)} \int_{s_{0}}^{t}(-\varepsilon) d \xi\right\}=0 .
$$

Hence, $\lim _{t \rightarrow \infty} E[V(t)]=0$.

Using the property $Y_{e}^{T}(t) Y_{e}(t) \leq V(t) / \lambda_{\text {min }}(P)$ yields

$$
\lim _{t \rightarrow \infty} E\left[Y_{e}^{T}(t) Y_{e}(t)\right]=\lim _{t \rightarrow \infty} E\left[x_{1}(t)-x_{j}(t)\right]^{2}=0 .
$$

On other hand, we have

$$
\begin{aligned}
E\left[x_{i}(t)-x_{j}(t)\right]^{2} \leq & 2 E\left[x_{i}(t)-x_{1}(t)\right]^{2} \\
& +2 E\left[x_{1}(t)-x_{j}(t)\right]^{2} .
\end{aligned}
$$

Finally,

$$
\lim _{t \rightarrow \infty} E\left[x_{i}(t)-x_{j}(t)\right]^{2}=0 .
$$

Therefore, the consensus protocol (4) can achieve weak mean square convergence.

A remark can be driven on condition (14), that is because the time-varying consensus gain $\alpha(t)$ is positive and vanishing at infinity, then, it is sufficient to show that $-2 l I_{n-1}+$ $2 P Q \ll 0$ which implies that $-2 l I_{n-1}+2 P Q-\alpha(t) I_{n-1} \ll 0$ for any positive value of $\alpha(t)$.

Let now the function $f(t, x)$ be linear in $x$ and denote this time by $\tilde{y}_{1}(t)=\pi X(t)$, and then

$$
\begin{aligned}
\pi F(t, X)= & f(t, \pi X)=f\left(t, \tilde{y}_{1}\right), \\
f\left(t, x_{1}\right)-f\left(t, x_{i}\right) & =f\left(t, x_{1}-x_{i}\right) \\
& =f\left(t, \pi X-\pi X+x_{1}-x_{i}\right) \\
& =f\left(t, \tilde{y}_{1}-\tilde{y}_{1}+y_{i}\right) \\
& =f\left(t, \tilde{y}_{1}\right)-f\left(t, \tilde{y}_{1}-y_{i}\right) .
\end{aligned}
$$

Hence, $S F(t, X)$ can be written in this case as

$$
\begin{aligned}
S F(t, X)= & {\left[f\left(t, \tilde{y}_{1}\right), f\left(t, \tilde{y}_{1}\right)-f\left(t, \tilde{y}_{1}-y_{2}\right), \ldots,\right.} \\
& \left.f\left(t, \tilde{y}_{1}\right)-f\left(t, \tilde{y}_{1}-y_{n}\right)\right]^{T} \\
= & {\left[f\left(t, \tilde{y}_{1}\right), F_{e}\left(t, Y_{e}\right)^{T}\right]^{T} . }
\end{aligned}
$$

And system (13) becomes

$$
\begin{aligned}
d \widetilde{y}_{1}(t)= & f\left(t, \widetilde{y}_{1}\right) d t+\alpha(t) \pi M d W(t), \\
d Y_{e}(t)= & F_{e}\left(t, Y_{e}\right) d t-\alpha(t) E L R Y_{e}(t) d t \\
& +\alpha(t) E M d W(t) .
\end{aligned}
$$

By following the same previous proof, we obtain $\lim _{t \rightarrow \infty} E\left[Y_{e}^{T}(t) Y_{e}(t)\right]=0$.

Now, from system (36), we have

$$
d \widetilde{y}_{1}(t)=f\left(t, \tilde{y}_{1}\right) d t+\alpha(t) \pi M d W(t) .
$$

Applying the Itô formula to the function $\lambda_{\max }(P) \tilde{y}_{1}^{2}(t)$ gives

$$
\begin{aligned}
d\left(\lambda_{\max }(P) \tilde{y}_{1}^{2}(t)\right)= & 2 \lambda_{\max }(P) \tilde{y}_{1}(t) f\left(t, \tilde{y}_{1}\right) d t \\
& +\lambda_{\max }(P) \alpha^{2}(t)\left((\pi M)(\pi M)^{T}\right) d t \\
& +2 \lambda_{\max }(P) \alpha(t) \tilde{y}_{1}(t) \pi M d W(t) .
\end{aligned}
$$

By Assumptions 4, we have

$$
\begin{aligned}
& 2 \tilde{y}_{1}(t) \lambda_{\max }(P)\left[f\left(t, \tilde{y}_{1}(t)\right)-k \tilde{y}_{1}(t)+k \tilde{y}_{1}(t)\right] \\
& \quad \leq\left[-2 l+2 \lambda_{\max }(P) k\right] \tilde{y}_{1}^{2}(t) .
\end{aligned}
$$


Then,

$$
\begin{aligned}
d\left(\lambda_{\max }(P) \tilde{y}_{1}^{2}(t)\right) \leq & {\left[-2 l+2 \lambda_{\max }(P) k\right] \tilde{y}_{1}^{2}(t) d t } \\
& +\lambda_{\max }(P) \alpha^{2}(t)\left((\pi M)(\pi M)^{T}\right) d t \\
& +2 \lambda_{\text {max }}(P) \alpha(t) \widetilde{y}_{1}(t) \pi M d W(t) .
\end{aligned}
$$

Since the matrix $Q$ could be positive definite matrix or negative definite matrix, as mentioned previously in Remark 5, by choosing $k$ as the largest eigenvalue of $Q$ and by the property of $\alpha(t)$, condition (14) ensures that $\left(-2 l+2 \lambda_{\max }(P) k\right)<0$.

Taking $\varepsilon=-2 l+2 \lambda_{\max }(P) k$ and following the same steps as these used to prove $\lim _{t \rightarrow \infty} E\left[Y_{e}^{T}(t) Y_{e}(t)\right]=0$, we can get

$$
\begin{aligned}
E\left[\widetilde{y}_{1}(t)\right]^{2} \leq & \tilde{y}_{1}^{2}(0) \exp \left\{\frac{1}{\lambda_{\max }(P)} \int_{0}^{t}(-\varepsilon) d s\right\} \\
& +\left(\lambda_{\max }(P)(\pi M)(\pi M)^{T}\right) \\
& \times \int_{0}^{t} \alpha^{2}(s) \exp \left\{\frac{1}{\lambda_{\max }(P)} \int_{s}^{t}(-\varepsilon) d \xi\right\} d s .
\end{aligned}
$$

From (41), we can deduce that $\lim _{t \rightarrow \infty} E\left[\widetilde{y}_{1}(t)\right]^{2}=0<\infty$.

This implies that $\tilde{y}_{1}^{2}(t)$ is a martingale and, by the martingale converge theorem [45, Theorem 4.3], $\widetilde{y}_{1}(t)$ converges in mean square to a random variable $y^{*}$ as time goes on. Thus,

$$
\lim _{t \rightarrow \infty} E\left[\widetilde{y}_{1}(t)-y^{*}\right]^{2}=0 .
$$

On other hand, we have

$$
\begin{aligned}
E\left[X(t)-1_{n} \otimes y^{*}\right]^{2}= & E\left[S^{-1} Y(t)-1_{n} \otimes y^{*}\right]^{2} \\
= & E\left[1_{n} \otimes \widetilde{y}_{1}(t)+R Y_{e}(t)-1_{n} \otimes y^{*}\right]^{2} \\
\leq & 2 E\left[1_{n} \otimes\left(\widetilde{y}_{1}(t)-y^{*}\right)\right]^{2} \\
& +2 \operatorname{tr}\left(R R^{T}\right) E\left[Y_{e}^{T}(t) Y_{e}(t)\right] .
\end{aligned}
$$

Therefore, $\lim _{t \rightarrow \infty} E\left[X(t)-1_{n} \otimes y^{*}\right]^{2} \leq 0$.

Hence, all the agents in the team converge in mean square to the same random variable $y^{*}$, and the proof is complete.

In what follows, we will study the case when agents receive information without measurement noises.

Let $\eta_{j i}(t)=0$; the consensus protocol (4) can be rewritten as

$$
u_{i}(t)=\alpha(t)\left[\sum_{j \in \mathscr{N}_{i}} a_{i j}\left(x_{j}(t)-x_{i}(t)\right)\right], \quad i=1,2, \ldots, n .
$$

The closed loop system (6) is reduced to

$$
\dot{X}(t)=F(t, X)+\alpha(t)(-L) X(t),
$$

and (36) is reduced to

$$
\begin{gathered}
\dot{\tilde{y}}_{1}(t)=f\left(t, \tilde{y}_{1}\right), \\
\dot{Y}_{e}(t)=F_{e}\left(t, Y_{e}\right)-\alpha(t) E L R Y_{e}(t) .
\end{gathered}
$$

Corollary 7. If Assumptions 3 and 4 hold, then, with consensus protocol (4), the single-integrator multiagent systems (2) achieve asymptotic convergence under directed fixed topology for any initial condition if

$$
-2 l I_{n-1}+2 P Q-\alpha(t) I_{n-1} \ll 0 .
$$

Proof. Choose the Lyapunov function candidate as

$$
V(t)=Y_{e}^{T}(t) P Y_{e}(t),
$$

where $P$ is a positive definite matrix that satisfies

$$
(-E L R) P+P(-E L R)^{T}=-I_{n-1} .
$$

By the derivation of $V(t)$ with respect to time along the trajectory $Y_{e}(t)$, we get

$$
\begin{aligned}
\dot{V}(t)= & 2 Y_{e}^{T}(t) P\left[F_{e}\left(t, Y_{e}\right)+Q Y_{e}(t)-Q Y_{e}(t)\right] \\
& +\alpha(t) Y_{e}^{T}(t)\left[(-E L R) P+P(-E L R)^{T}\right] Y_{e}(t) .
\end{aligned}
$$

Similar to the proof of Theorem 6, by using Assumption 4 and the fact that $(-E L R)$ is a stable matrix, we get

$$
\dot{V}(t) \leq Y_{e}^{T}(t)\left[-2 l I_{n-1}+2 P Q-\alpha(t) I_{n-1}\right] Y_{e}(t) .
$$

It can be seen that if $-2 l I_{n-1}+2 P Q-\alpha(t) I_{n-1} \ll 0$

$$
\dot{V}(t) \leq \frac{-\varepsilon}{\lambda_{\max }(P)} V(t),
$$

which implies that $\dot{V}(t)<0$, for any $Y_{e}(t) \neq 0$, and the proof is complete.

Remark 8. From the proof of Corollary 7, one can see that the two conditions on $\alpha(t), \int_{0}^{\infty} \alpha(t) d t=\infty$, and $\int_{0}^{\infty} \alpha^{2}(t) d t<\infty$ are not necessary.

Remark 9. In the noise-free case, it is easy also to show that the group can achieve asymptotic convergence in mean square, and, after some manipulations, we can get

$$
E[V(t)] \leq E[V(0)] \exp \left\{\frac{1}{\lambda_{\max }(P)} \int_{0}^{t}(-\varepsilon) d s\right\},
$$

which implies that $\lim _{t \rightarrow \infty} E[V(t)]=0$.

Remark 10. In the noisy case, when $t \rightarrow \infty$, the consensus is achieved in mean square, and from (37), we have

$$
\begin{aligned}
E\left[y^{*}\right]= & \tilde{y}_{1}(0)+E \int_{0}^{\infty} f\left(s, \widetilde{y}_{1}\right) d s=\tilde{y}_{1}(0) \\
& +\int_{0}^{\infty} f\left(s, E\left[\tilde{y}_{1}(s)\right]\right) d s=\sum_{1}^{n} \pi_{i} x_{i}(0) \\
& +\int_{0}^{\infty} f\left(s, \sum_{1}^{n} \pi_{i} E\left[x_{i}(s)\right]\right) d s .
\end{aligned}
$$




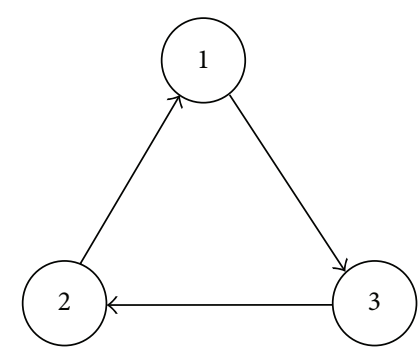

FIGURE 1: Digraph $\mathscr{G}_{1}$ has a spanning tree.

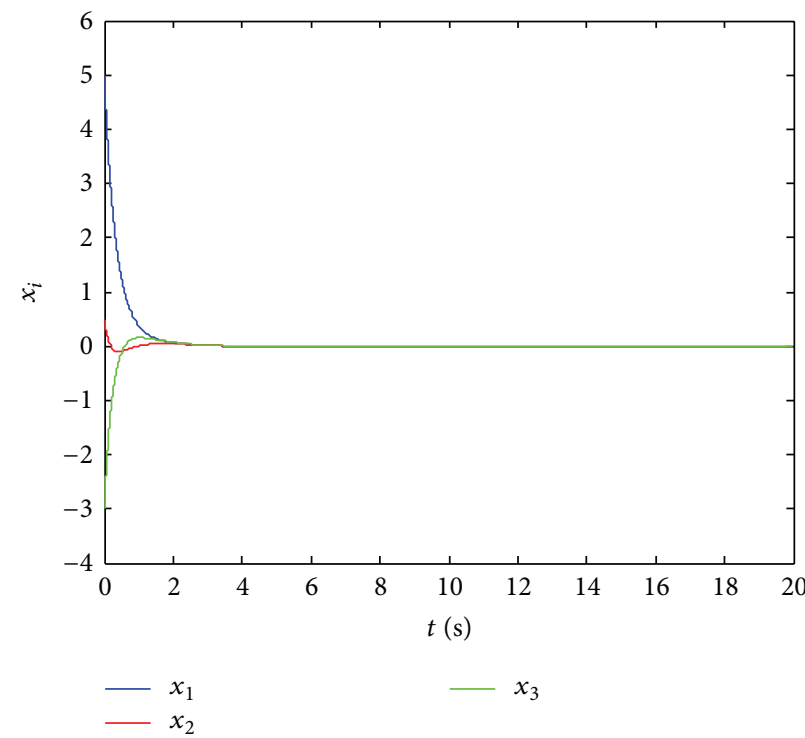

(a) With $\alpha(t)=1$

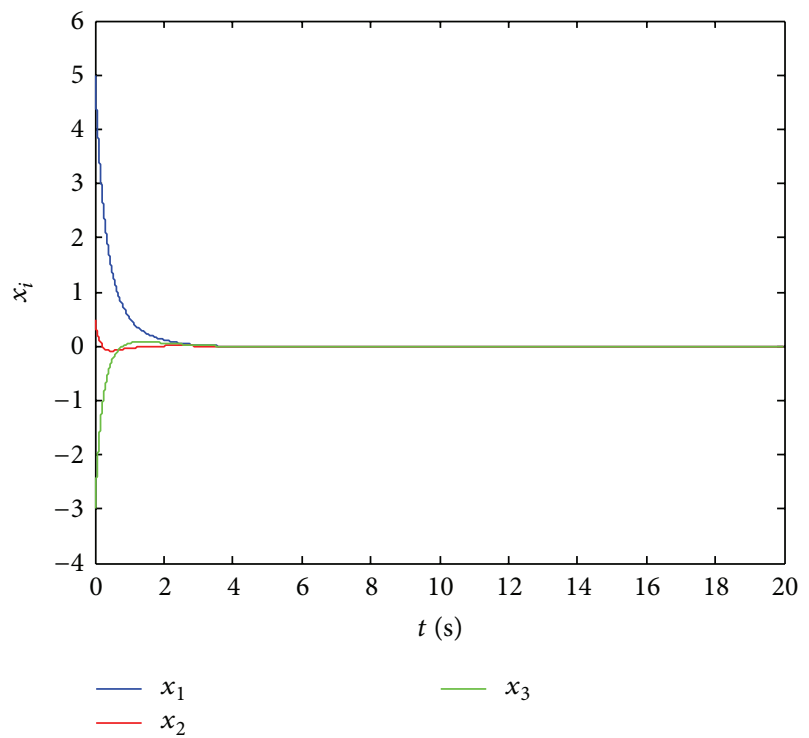

(b) With $\alpha(t)=1 /(1+t)$

Figure 2: States of the three agents under digraph $\mathscr{G}_{1}$ without measurement noises.

It is obvious that the mathematical expectation of the final consensus is a time-varying value.

In the free-noise case, when the consensus is asymptotically achieved, we have $\lim _{t \rightarrow \infty} Y_{e}(t)=0$ and, from (46), it is clear that the final consensus is time-varying and has the dynamic $\dot{x}=f(t, x)$ with initial state $x(0)=\sum_{1}^{n} \pi_{i} x_{i}(0)$.

Remark 11. In the absence of the inherent nonlinear dynamics, the control law (4) still guarantees the mean square convergence in digraphs having spanning three and it does not require that the digraph is balanced; this result makes the protocol proposed in [28] for unbiased mean square convergence in single-integrator dynamics under balanced digraphs a special case of our protocol when $\pi=[1 / n, 1 / n, \ldots, 1 / n]$ and $E\left[y^{*}\right]=(1 / n) \sum_{1}^{n} x_{i}(0)$.

\section{Simulations}

In this section, two examples are given to demonstrate the validity of the consensus protocols (4) and (44).

We assume there are three agents $(n=3)$ in the team. The topology among the three agents is fixed and has a spanning tree as shown in Figure 1. The inherent nonlinear dynamics is given by $f(t, x)=x[-2+\sin (t)]$. With digraph
$\mathscr{G}_{1}$, the positive definite matrix $P$ satisfying (16) and (49) is $\left[\begin{array}{ccc}0.2778 & 0.05556 \\ 0.05556 & 0.4444\end{array}\right]$, the matrix $Q$ is chosen as $P^{-1}$, and the constant $\varepsilon$ satisfying (14) and (47) is given by $\varepsilon=0.1$. The initial states of the three agents are $5,0.5$, and -3 , respectively.

Example 1. We first consider the case of noise-free measurement, it can be seen from Figure 2 how the team under consensus protocol (44) asymptotically converges to a common value that has the dynamics $\dot{x}=x[-2+\sin (t)]$ whether $\alpha(t)=1, t \geq 0$ Figure 2(a) or $\alpha(t)=1 /(1+t), t \geq 0$ Figure 2(b).

Example 2. In this example, we consider the case when the communication is corrupted by measurement noises. Two scenarios are considered. In the first one, we let $\sigma=\sigma_{13}=$ $\sigma_{21}=\sigma_{32}=0.1$ and in the second one, $\sigma=\sigma_{13}=\sigma_{21}=$ $\sigma_{32}=1$. Each scenario is simulated for both $\alpha(t)=1, t \geq 0$ and $\alpha(t)=1 /(1+t), t \geq 0$ under consensus protocol (4). Figure 3 depicts the performance of the first scenario as time goes on and it can be seen that the closed loop has a random dynamics for $\alpha(t)=1$ Figure 3(a), and convergent to random value in mean square having the mathematical expectation (54) for $\alpha(t)=1 /(1+t), t \geq 0$ Figure 3(b). Figure 4 shows 


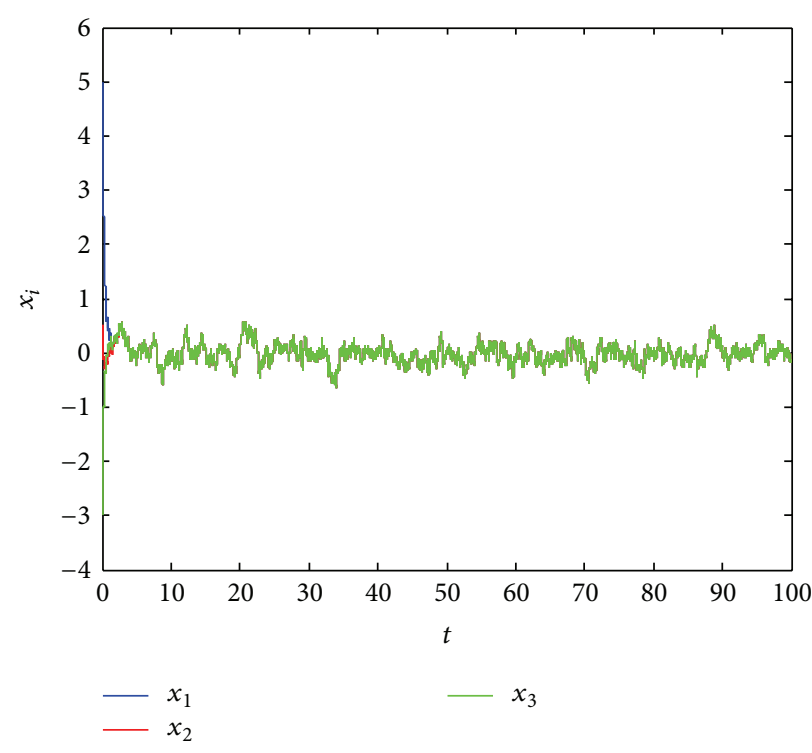

(a) With $\alpha(t)=1$

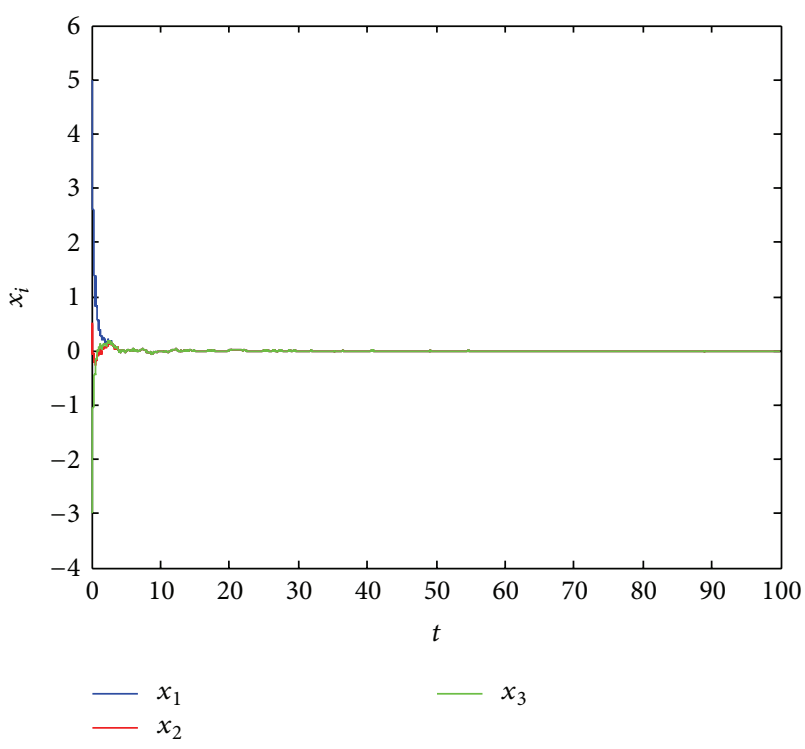

(b) With $\alpha(t)=1 /(1+t)$

FIgURE 3: States of the three agents under digraph $\mathscr{G}_{1}$ with noise intensity $\sigma=0.1$.

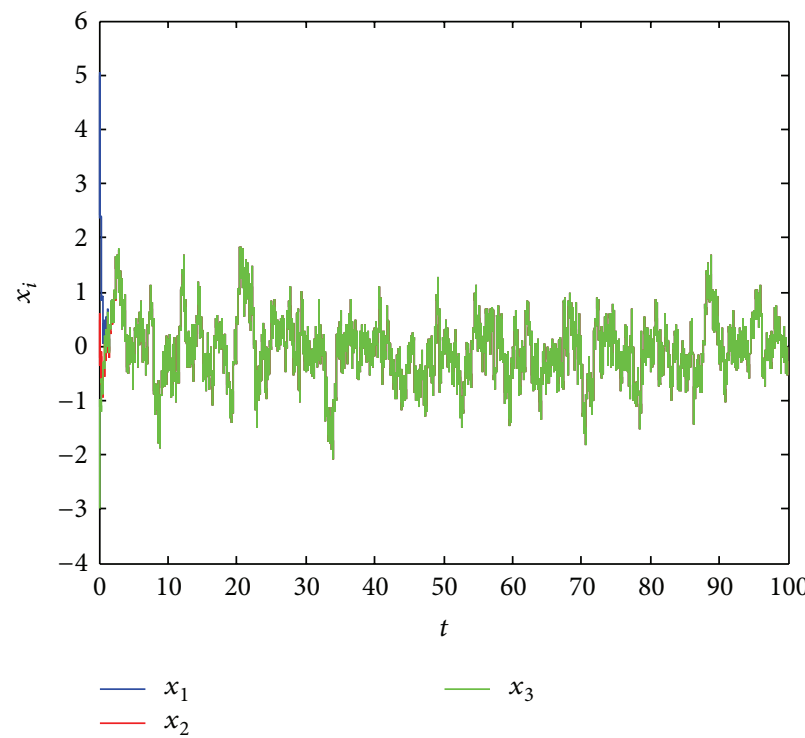

(a) With $\alpha(t)=1$

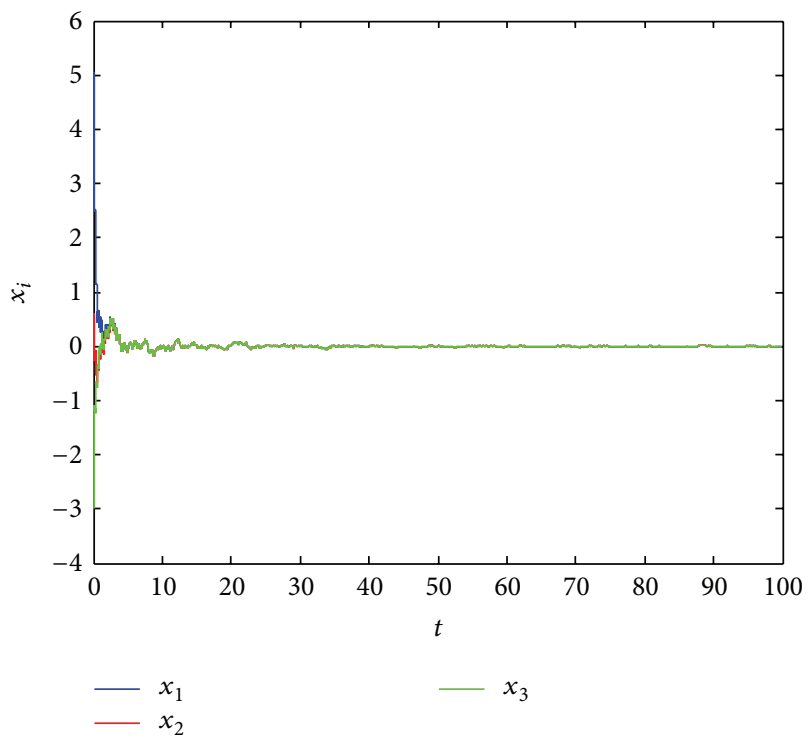

(b) With $\alpha(t)=1 /(1+t)$

FIgURE 4: States of the three agents under digraph $\mathscr{G}_{1}$ with noise intensity $\sigma=1$.

how that our consensus protocol (4) is effective even with considerable noise intensity and asymptotically solves the consensus problem in mean square with $\alpha(t)=1 /(1+t), t \geq$ 0 . In contrast to Figure 2(b), where the asymptotic consensus is achieved with a deterministic final consensus due to the absence of noises, the final consensus in Figures 3(b) and 4(b) is random.

\section{Conclusion}

In this paper, we have addressed the consensus problem of single-integrator multiagent systems with inherent nonlinear dynamics and measurement noises under directed fixed topologies in a unified cooperative control. Conditions to guarantee asymptotic stability in mean square in presence of measurement noises and asymptotic stability for the noisefree case were derived. Numerical simulations to validate the effectiveness of our approach were handled and through which we validated that under noisy communications the agents' states in the team converge in mean square to a random value and in the free-noise case the agents' states converge to a time-varying value that has dynamics similar to the nonlinear inherent dynamics. In our future work, we will investigate the case of switching topologies. We expect 
that some existing works on switched stochastic systems like $[46,47]$ can be helpful in resolving this problem.

\section{Conflict of Interests}

The authors declare that there is no conflict of interests regarding the publication of this paper.

\section{Acknowledgments}

This work was supported by the National Nature Science Foundation of P. R China under Grant 61321002 and the Program for Changjiang Scholars and Innovative Research Team in the university under Grant IRT 1208.

\section{References}

[1] W. Burgard, M. Moors, D. Fox, R. Simmons, and S. Thrun, "Collaborative multi-robot exploration," in Proceedings of the IEEE International Conference on Robotics and Automation (ICRA '00), pp. 476-481, San Francisco, Calif, USA, April 2000.

[2] C. J. Taylor, "VideoPlus: a method for capturing the structure and appearance of immersive environments," IEEE Transactions on Visualization and Computer Graphics, vol. 8, no. 2, pp. 171$182,2002$.

[3] A. W. Stroupe, M. C. Martin, and T. Balch, "Distributed sensor fusion for object position estimation by multi-robot systems," in Proceedings of the IEEE International Conference on Robotics and Automation (ICRA '01), pp. 1092-1098, Seoul, Korea, May 2001.

[4] T. Sugar and V. Kumar, "Control and coordination of multiple mobile robots in manipulation and material handling tasks," in Experimental Robotics VI, vol. 250 of Lecture Notes in Control and Information Sciences, pp. 15-24, Springer, Berlin, Germany, 2000.

[5] P. Ögren, E. Fiorelli, and N. E. Leonard, "Cooperative control of mobile sensor networks: adaptive gradient climbing in a distributed environment," IEEE Transactions on Automatic Control, vol. 49, no. 8, pp. 1292-1302, 2004.

[6] W. Ren and R. W. Beard, "Consensus seeking in multiagent systems under dynamically changing interaction topologies," IEEE Transactions on Automatic Control, vol. 50, no. 5, pp. 655661, 2005.

[7] W. Ren and E. Atkins, "Distributed multi-vehicle coordinated control via local information exchange," International Journal of Robust and Nonlinear Control, vol. 17, no. 10-11, pp. 1002-1033, 2007.

[8] Y. Cao and W. Ren, "Distributed coordinated tracking with reduced interaction via a variable structure approach," IEEE Transactions on Automatic Control, vol. 57, no. 1, pp. 33-48, 2012.

[9] Y. Cao and W. Ren, "Distributed coordinated tracking via a variable structure approach-part I: consensus tracking," in Proceedings of the American Control Conference (ACC '10), pp. 4744-4749, Baltimore, Md, USA, July 2010.

[10] W. Ren, "Multi-vehicle consensus with a time-varying reference state," Systems \& Control Letters, vol. 56, no. 7-8, pp. 474-483, 2007.

[11] G. Chen, X. Wang, X. Li, and J. Lü, "Some recent advances in complex networks synchronization," in Recent Advances in Nonlinear Dynamics and Synchronization, vol. 254 of Studies in Computational Intelligence, pp. 3-16, Springer, Studies in Computational Intelligence, 2009.

[12] W. Yu, G. Chen, and M. Cao, "Consensus in directed networks of agents with nonlinear dynamics," IEEE Transactions on Automatic Control, vol. 56, no. 6, pp. 1436-1441, 2011.

[13] K. Liu, G. Xie, W. Ren, and L. Wang, "Consensus for multiagent systems with inherent nonlinear dynamics under directed topologies," Systems and Control Letters, vol. 62, no. 2, pp. 152162, 2013.

[14] Y. Cao, W. Ren, F. Chen, and G. Zong, "Finite-time consensus of multi-agent networks with inherent nonlinear dynamics under an undirected interaction graph," in Proceedings of the American Control Conference (ACC '11), pp. 4020-4025, San Francesco, Calif, USA, July 2011.

[15] Y. Cao and W. Ren, "Finite-time consensus for single-integrator kinematics with unknown inherent nonlinear dynamics under a directed interaction graph," in Proceedinsg of the American Control Conference (ACC '12), pp. 1603-1608, Fairmont Queen Elizabeth, Montréal, Canada, June 2012.

[16] Y. Cao and W. Ren, "Finite-time consensus for second-order multi-agent networks with inherent nonlinear dynamics under an undirected fixed graph," in Proceedings of the 50th IEEE Conference on Decision and Control and European Control Conference (CDC-ECC '11), pp. 3452-3457, Orlando, Fla, USA, December 2011.

[17] W. Yu, G. Chen, M. Cao, and J. Kurths, "Second-order consensus for multiagent systems with directed topologies and nonlinear dynamics," IEEE Transactions on Systems, Man, and Cybernetics Part B: Cybernetics, vol. 40, no. 3, pp. 881-891, 2010.

[18] Z. Li, X. Liu, and M. Fu, "Global consensus control of Lipschitz nonlinear multi-agent systems," in Proceedings of the 18th IFAC World Congress Part 1, vol. 18, pp. 10056-10061, Milano, Italy, September 2011.

[19] G. G. Wen, A. Rahmani, and Y. G. Yu, "Consensus tracking for multi-agent systems with nonlinear dynamics under fixed communication topologies," in Proceedings of the World Congress on Engineering and Computer Science, San Francisco, Calif, USA, October 2011.

[20] Y. Zhang, Y. Yang, Y. Zhao, and G. Wen, "Finite-time consensus tracking for nonlinear multi-agent systems under undirected and directed communication topologies," in Proceedings of the 31st Chinese Control Conference (CCC '12), pp. 6214-6219, Hefei, China, July 2012.

[21] Q. Song, J. D. Cao, and W. W. Yu, "Second-order leaderfollowing consensus of nonlinear multi-agent systems via pinning control," Systems \& Control Letters, vol. 59, no. 9, pp. 553$562,2010$.

[22] H. Su, G. Chen, X. Wang, and Z. Lin, "Adaptive secondorder consensus of networked mobile agents with nonlinear dynamics," Automatica, vol. 47, no. 2, pp. 368-375, 2011.

[23] H. Zhang and F. L. Lewis, "Adaptive cooperative tracking control of higher-order nonlinear systems with unknown dynamics," Automatica, vol. 48, no. 7, pp. 1432-1439, 2012.

[24] M. Huang and J. H. Manton, "Coordination and consensus of networked agents with noisy measurements: stochastic algorithms and asymptotic behavior," SIAM Journal on Control and Optimization, vol. 48, no. 1, pp. 134-161, 2009.

[25] M. Huang and J. H. Manton, "Stochastic approximation for consensus seeking: mean square and almost sure convergence," in Proceedings of the 46th IEEE Conference on Decision and Control (CDC '07), pp. 306-311, New Orleans, La, USA, December 2007. 
[26] M. Huang and J. H. Manton, "Stochastic consensus seeking with measurement noise: convergence and asymptotic normality," in Proceedings of the American Control Conference (ACC '08), pp. 1337-1342, Seattle, Wash, USA, June 2008.

[27] T. Li and J.-F. Zhang, "Consensus conditions of multi-agent systems with time-varying topologies and stochastic communication noises," IEEE Transactions on Automatic Control, vol. 55, no. 9, pp. 2043-2057, 2010.

[28] T. Li and J.-F. Zhang, "Mean square average-consensus under measurement noises and fixed topologies: necessary and sufficient conditions," Automatica, vol. 45, no. 8, pp. 1929-1936, 2009.

[29] J. Liu, X. Liu, W.-C. Xie, and H. Zhang, "Stochastic consensus seeking with communication delays," Automatica, vol. 47, no. 12, pp. 2689-2696, 2011.

[30] L. Cheng, Z.-G. Hou, M. Tan, and X. Wang, "Necessary and sufficient conditions for consensus of double-integrator multiagent systems with measurement noises," IEEE Transactions on Automatic Control, vol. 56, no. 8, pp. 1958-1963, 2011.

[31] J. Hu and G. Feng, "Distributed tracking control of leaderfollower multi-agent systems under noisy measurement," Automatica, vol. 46, no. 8, pp. 1382-1387, 2010.

[32] Y. L. Shang, "Leader-following consensus problems with a time-varying leader under measurement noises," Advances in Dynamical Systems and Applications, vol. 6, no. 2, pp. 255-270, 2011.

[33] C. Ma, T. Li, and J. Zhang, "Consensus control for leaderfollowing multi-agent systems with measurement noises," Journal of Systems Science and Complexity, vol. 23, no. 1, pp. 35-49, 2010.

[34] S. Djaidja and Q. H. Wu, "Leader-following consensus of double-integrator multi-agent systems with noisy measurements," Accepted to International Journal of Control, Automation and Systems.

[35] Y.-H. Ni and X. Li, "Consensus seeking in multi-agent systems with multiplicative measurement noises," Systems and Control Letters, vol. 62, no. 5, pp. 430-437, 2013.

[36] S. Djaidja and Q. H. Wu, "Leaderless consensus seeking in multi-agent systems under multiplicative measurement noises and switching topologies," in Proceedings of the 33rd Chinese Control Conference, Nanjing, China, July 2014.

[37] S. Djaidja and Q. H. Wu, "Leader-following consensus for single-integrator multi-agent systems with multiplicative noises in directed topologies," International Journal of Systems Science, 2014.

[38] W. Ren, "Consensus seeking in multi-vehicle systems with a time-varying reference state," in Proceedings of the American Control Conference, pp. 717-722, New York, NY, USA, July 2007.

[39] J. Xiang, Y. Li, and W. Wei, "Global synchronization of directional networked systems with eventually dissipative nodes," IEEE Transactions on Circuits and Systems. I. Regular Papers, vol. 59, no. 6, pp. 1278-1289, 2012.

[40] W. Lu, X. Li, and Z. Rong, "Global stabilization of complex networks with digraph topologies via a local pinning algorithm," Automatica, vol. 46, no. 1, pp. 116-121, 2010.

[41] J. Qiu, J. Lu, J. Cao, and H. He, “Tracking analysis for general linearly coupled dynamical systems," Communications in Nonlinear Science and Numerical Simulation, vol. 16, no. 4, pp. 20722085, 2011.

[42] C. W. Wu, "Synchronization in arrays of coupled nonlinear systems with delay and nonreciprocal time-varying coupling,"
IEEE Transactions on Circuits and Systems II: Express Briefs, vol. 52, no. 5, pp. 282-286, 2005.

[43] B. Øksendal, Stochastic Differential Equations, An Introduction with Applications, Springer, 6th edition, 2003.

[44] A. N. Michel and R. K. Miller, Qualitative Analysis of Large Scale Dynamical Systems, Academic Press, New York, NY, USA, 1977.

[45] J. M. Steele, Stochastic Calculus and Financial Applications, Springer, 2001.

[46] L. Hou, G. Zong, Y. Wu, and Y. Cao, "Exponential $l_{2}-l_{\infty}$ output tracking control for discrete-time switched system with timevarying delay," International Journal of Robust and Nonlinear Control, vol. 22, no. 11, pp. 1175-1194, 2012.

[47] G. Zong, R. Wang, W. X. Zheng, and L. Hou, "Finite-time stabilization for a class of switched time-delay systems under asynchronous switching," Applied Mathematics and Computation, vol. 219, no. 11, pp. 5757-5771, 2013. 


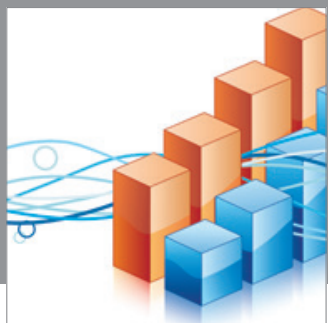

Advances in

Operations Research

mansans

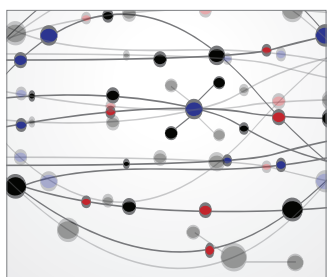

The Scientific World Journal
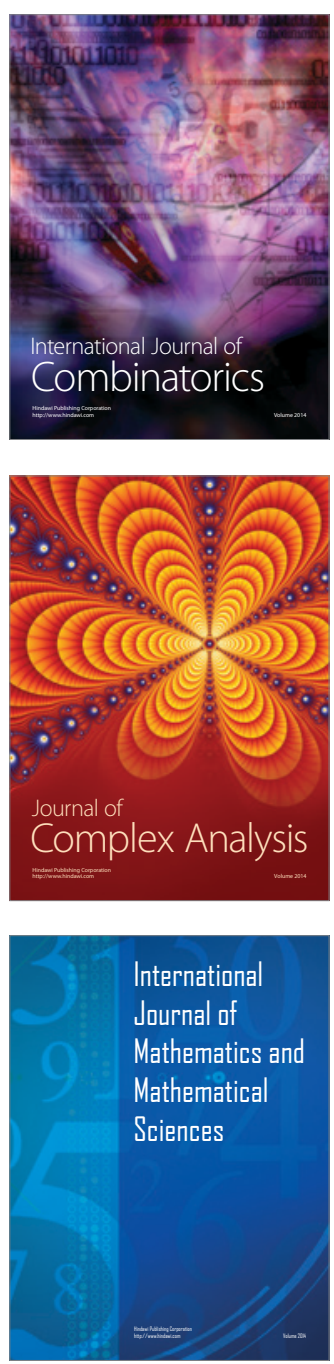
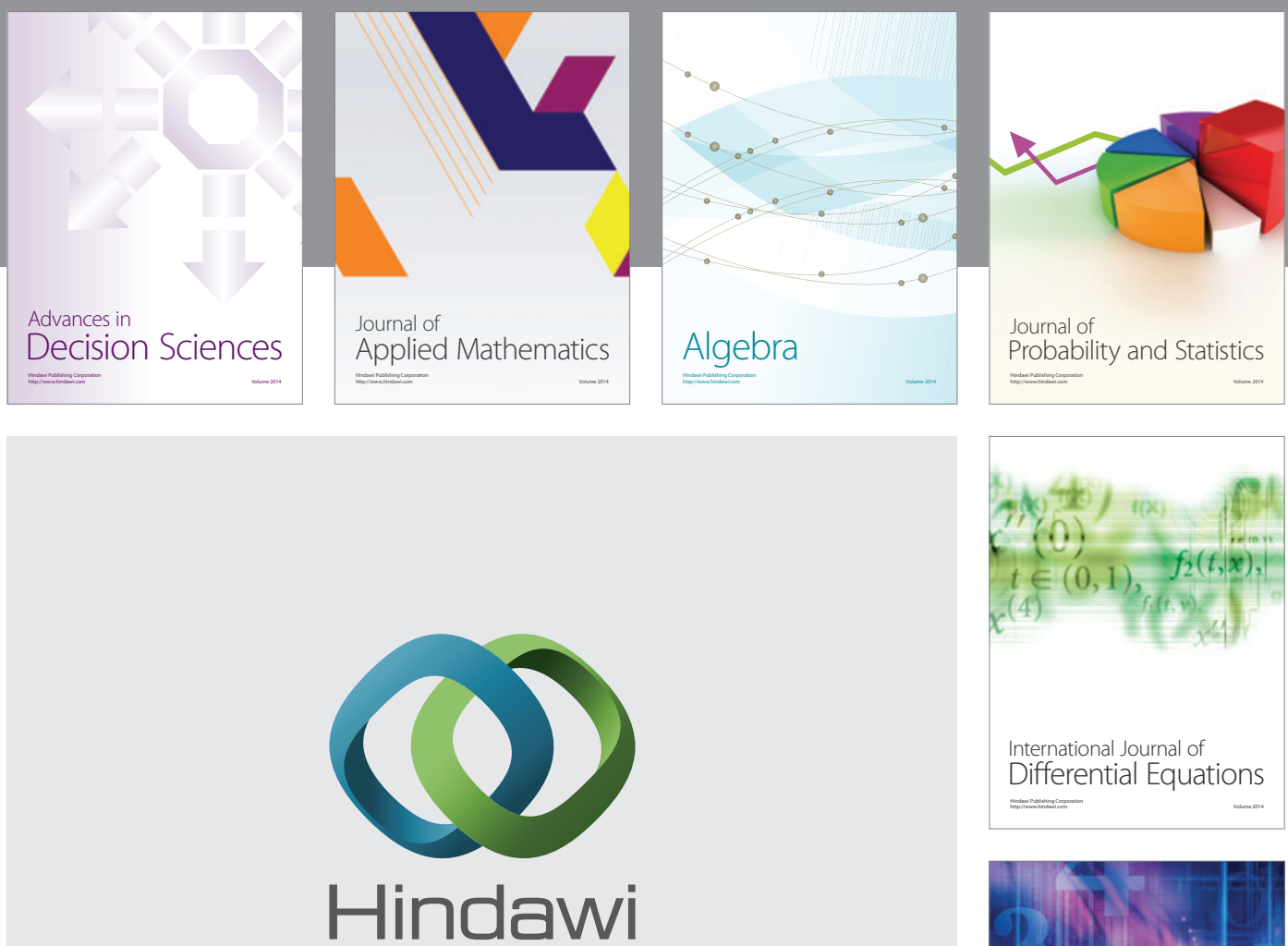

Submit your manuscripts at http://www.hindawi.com
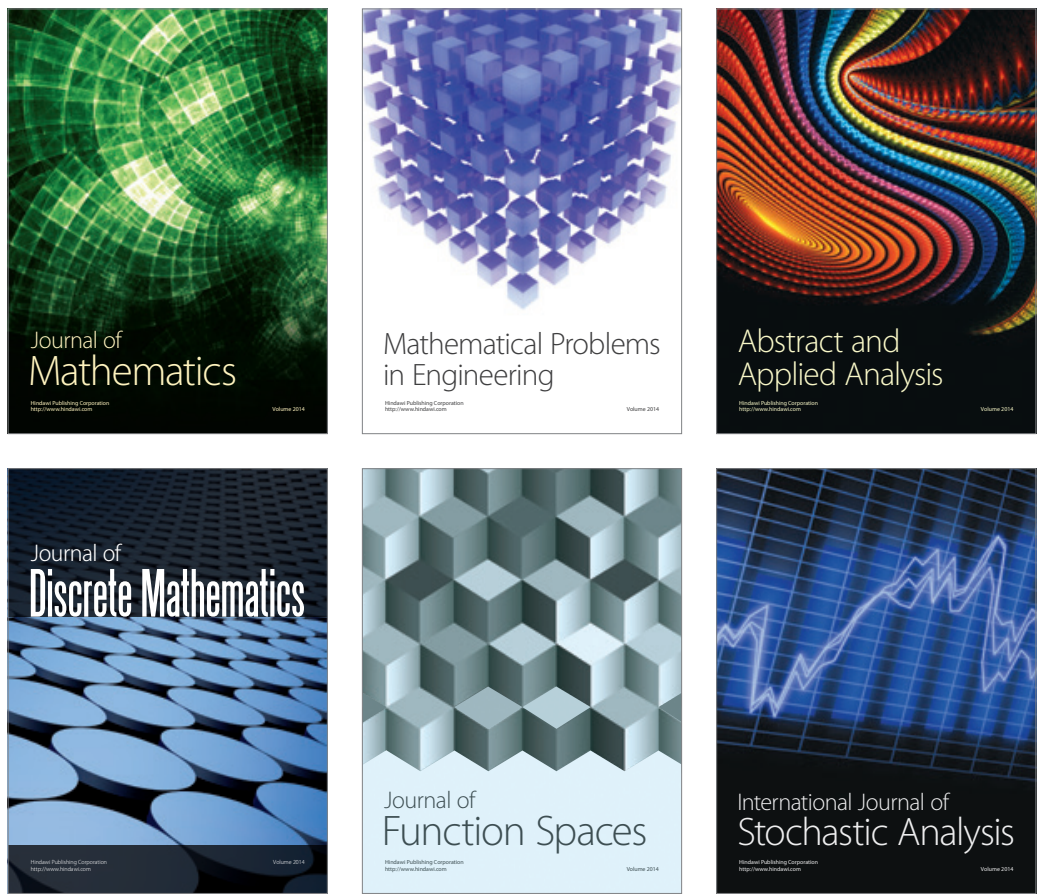

Journal of

Function Spaces

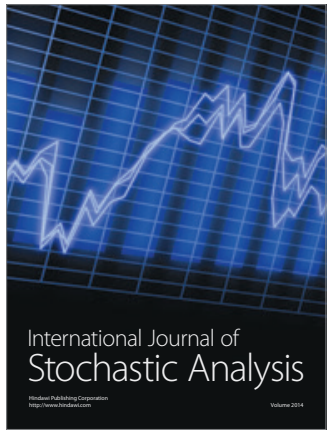

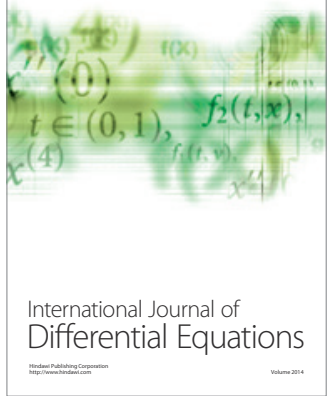
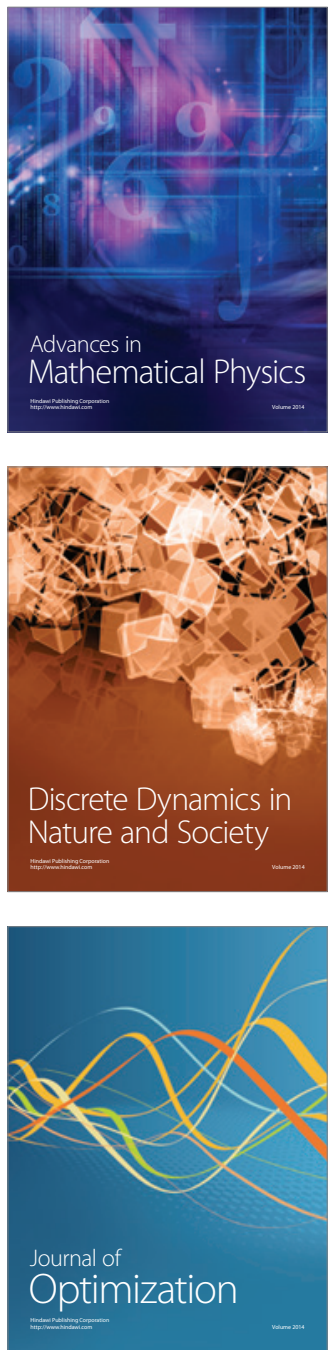